La historia de Zamba. Valoraciones ideológicas en el audiovisual infantil

Sabina Crivelli

Tram[p]as de la comunicación y la cultura (N. $\left.{ }^{\circ} 85\right)$, e044, 2020

ISSN 2314-274X | https://doi.org/10.24215/2314274xe044

http://perio.unlp.edu.ar/ojs/index.php/trampas

FPyCS | Universidad Nacional de La Plata

La Plata | Buenos Aires | Argentina

\title{
LA HISTORIA DE ZAMBA
}

\section{VALORACIONES IDEOLÓGICAS EN EL AUDIOVISUAL INFANTIL}

\author{
ZAMBA'S STORY: IDEOLOGICAL ASSESSMENTS IN CHILDREN'S AUDIOVISUAL
}

\author{
Sabina Crivelli \\ sabinacrivelli@gmail.com \\ https://orcid.org/0000-0003-3369-2376 \\ Instituto de Estudios Comunicacionales en Medios, \\ Cultura y Poder "Aníbal Ford" (INESCO) \\ Universidad Nacional de La Plata | Argentina
}

\begin{abstract}
Resumen
$\mathrm{El}$ artículo analiza valoraciones del producto audiovisual La asombrosa excursión de Zamba, impulsado por el Ministerio de Educación argentino durante el período 2010- 2015 y con circulación aun en 2016. El estudio se enmarca en una investigación sobre la circulación del programa de televisión infantil. Focaliza en la exploración de las mediaciones comunicacionales que se establecen en relación con los significados históricos, ideológicos, políticos, estéticos. A partir de situar la indagación en la circulación, se analizan algunas valoraciones de la prensa digital y de docentes de CABA sobre los modos narrativos y estéticos del producto.
\end{abstract}

Abstract

\begin{abstract}
The article analyzes evaluations of the audiovisual product La asombrosa excursión de Zamba, promoted by the Argentine Ministry of Education during the period 2010-2015 and with circulation still in 2016. The analysis is part of an investigation on the circulation of the children's television program. It focuses on the exploration of communicational mediations that are established in relation to historical, ideological, political and aesthetic meanings. Starting from placing the inquiry in circulation, some evaluations of digital press and CABA teachers about the narrative and aesthetic modes of the product are analyzed.
\end{abstract}

Palabras clave | audiovisual, Zamba, significaciones sociales, circulación Keywords | audiovisual, Zamba, social meanings, circulation 


\title{
LA HISTORIA DE ZAMBA
}

VALORACIONES IDEOLÓGICAS EN EL AUDIOVISUAL INFANTIL

\author{
Por Sabina Crivelli
}

\section{Zamba desde un análisis cultural}

El tiempo histórico político es quizás una de las marcas más fuertes en la circulación de las significaciones que se analizan en este trabajo. El dibujo animado argentino que tiene como personaje protagónico a Zamba se instaló desde 2010 en el marco del surgimiento del canal de televisión infantil Pakapaka, del Ministerio de Educación de la Nación. Tal vez, la iniciativa más firmemente orientada a disputar algunos aspectos de la hegemonía cultural. A partir de entender que los medios son simbólicos instituyentes, en esta investigación ${ }^{1}$ nos proponemos indagar sobre la incidencia y la tensión generada por la intervención cultural de una iniciativa del Estado a instancias de una política pública más amplia.

Desde 2009, encontramos que el gobierno nacional asumió como política de Estado la necesidad de impulsar la democratización de la comunicación. En ese marco, un año más tarde se puso al aire la señal de televisión infantil Pakapaka, que fue presentada como una opción innovadora para la infancia. Desde el inicio, el programa que aquí elegimos analizar fue el producto de mayor circulación del canal. La asombrosa excursión de Zamba irrumpió en la oferta de productos audiovisuales para disputar públicos a otros productos masivos con códigos culturales hegemónicos, a la vez que empezó a ser utilizado por los/as docentes en las escuelas primarias. 
Un aspecto a destacar es que entre 2010 y 2016 la circulación de este producto hizo aparecer en primer plano disputas sobre la historia argentina que parecerian actualizar conflictos del presente. La delimitación del periodo de estudio obedece a que desde esos primeros años fundacionales hasta 2015 las disputas y las tensiones se visibilizaron en la prensa digital. Lo mismo sucedió en 2016, durante el primer año del gobierno de Mauricio Macri. Luego, ya no aparece como un terreno de conflicto o, al menos, este parece haberse diluido en la prensa digital, probablemente por su menor circulación.

Desde la perspectiva materialista de la cultura (Williams, 2000) aquí adoptada, se reconocen los procesos de producción-circulación-recepción de la cultura para estudiar las sedimentaciones históricas de sentido que se actualizan en las valoraciones y en las representaciones mediáticas de La asombrosa excursión de Zamba. Desde un punto de vista teórico, se asume la perspectiva de los estudios culturales, la cual se interesa por los modos de organización simbólica de las prácticas y cuyo foco central se ubica en las disimetrías (Hall, 1980).

De este modo, se busca analizar los procesos y las prácticas concretas desde las cuales un contexto se construye como una organización de relaciones que entendemos en tanto coyunturas. Es decir, como una "formación social fracturada y conflictual, a lo largo de múltiples ejes, planos y escalas, en una búsqueda constante de equilibrios provisorios o estabilidades estructurales mediante una variedad de prácticas y procesos de lucha y negociación" (Grossberg, 2006, p. 56). Así entendida, la realidad social es concebida como configuración de relaciones constantemente abiertas a la rearticulación.

Desde el coyunturalismo planteado por Stuart Hall (1984) y retomado por Lawrence Grossberg (2006), se trata de atender a la configuración cambiante de fuerzas que, ocasionalmente, busca, y algunas veces alcanza, un equilibrio o arreglo temporal. De este modo, se hace énfasis en la constante reconfiguración de un campo que produce solo estabilidades temporales y cuyo análisis involucra el mapeo de la redisposición de elementos en una configuración. En el caso de Zamba, este mapeo incluye el relevamiento del producto, la descripción del campo de la producción, los públicos del sector educativo entre los que circuló y que se apropiaron del mismo, y las valoraciones de otros actores que se pronunciaron en el espacio público. 
Para ello, se despliega una estrategia metodológica de corte cualitativo que contempla, por un lado, un análisis semiótico sobre los sentidos alojados en los principales signos ideológicos (Volóshinov, 2009 [1929]) presentes en el corpus de notas periodísticas relevadas y, por otro, el abordaje de las entrevistas etnográficas (Guber, 2009 [1991]) realizadas a informantes clave vinculados con el proceso de producción entre 2010 y 2015.

La asombrosa excursión de Zamba se posicionó como un producto masivo, y asî fue reconocido por diferentes actores legitimados en el campo ${ }^{2}$ cultural, que desde lecturas oposicionales, negociadas o dominantes, en términos de Stuart Hall (1980), lo vieron y establecieron alguna valoración. De esto último, se desprenden los interrogantes que motorizan el presente análisis. ¿Cuáles fueron las valoraciones del producto que circularon en los principales medios nacionales? ¿Qué dinámicas ideológicas de negociación y que disputas de sentido se constituyeron entre periodistas, académicos/as y docentes frente a lo propuesto por La asombrosa excursión de Zamba?

Se recorren en este trabajo las valoraciones que circularon en medios de prensa de alcance nacional sobre los modos narrativos y estéticos del producto en tensión con la propuesta de un abordaje del relato histórico. En este camino, no se abordará la singularidad de cada hecho histórico sino la valoración en general del producto en relación con la historia.

Para llevar adelante el análisis, se relevaron las publicaciones digitales realizadas entre 2010 y 2016 por los cuatro medios que mayor seguimiento dieron al tema: La Nación, Clarín, Página/12 e Infobae. Asimismo, fueron entrevistados catorce docentes de la Ciudad Autónoma de Buenos Aires (CABA) durante 2016, cuando Zamba si bien como producto transitaba otra etapa, la anterior aún estaba muy presente.

En los intersticios de estas valoraciones sobre un producto que supo ubicarse en el campo de disputa del mercado simbólico es posible identificar algunas categorias que dan cuenta de los modos en los que aparece la sedimentación de la hegemonía. Asimismo, también podremos advertir cómo algunas de las disputas ideológicas en el campo de la cultura parecerían enunciarse en términos estéticos. 
Estos argumentos se enmarcan, a su vez, en la asunción de que en los procesos de la hegemonía cultural intervienen múltiples instancias de producción y de mediación de sentidos. De ahí que elijamos tanto las valoraciones del periodismo o de expertos/as, que podrian considerarse intérpretes del fenómeno masivo Zamba, como de los/as docentes que, en tanto consumidores del producto, revisten un especial interés por utilizarlo, además, con los públicos infantiles en la institución escolar.

\section{El contexto de surgimiento y de circulación}

Desde una perspectiva sociosemiótica de la cultura, entendemos que los medios de comunicación masiva, que incluyen la televisión, la radio, la prensa, el cine y el ciberespacio, donde se constituyen las redes sociales, resultan centrales para entender la transformación de los códigos culturales y las batallas que en ellos se libran. Precisamente, desde un planteo afin a esta idea, en la Argentina, entre 2009 y 2015, se produjo una intensa discusión en torno a los valores hegemónicos en este campo. En el marco de un proceso denominado de democratización y de desmonopolización de la comunicación audiovisual, se implementaron diversas politicas públicas a partir de demandas que tenían, al menos, una década entre diversos sectores y organizaciones sociales y politicas. La necesidad de ampliar la participación de actores y las representaciones en la producción audiovisual estaba instalada. Pero, además, en este breve lapso de tiempo estas demandas, más allá de las discusiones sobre los aciertos o los errores, pudieron ser reconocidas y plasmadas en varias políticas públicas concretas.

Los públicos infantiles no solo no quedaron exentos sino que tal vez la principal iniciativa que de forma planificada se orientó a una disputa de ciertos aspectos de la hegemonía cultural estuvo destinada, justamente, a la infancia. En este marco surgió la señal televisiva Pakapaka, presentada como una opción innovadora para los públicos infantiles que, desde un primer momento, fueron concebidos y nombrados como ciudadanos en oposición a lo que se enunció como un abordaje en tanto consumidores.

En la breve masividad de su circulación, La asombrosa excursión de Zamba tuvo diferentes momentos. Surgió en 2010, en el contexto del Bicentenario de la Argentina, cuando el Ministerio de Educación de la Nación encargó contenidos 
para explicar este acontecimiento histórico al público infantil en cuatro capítulos a emitirse desde la pantalla del canal educativo Encuentro. La productora contratada para este fin -El perro en la luna, de Sebastián Mignona-, ubicada en la Ciudad Autónoma de Buenos Aires, creó el personaje de Zamba, un niño inquieto que asiste a una escuela pública y es oriundo de Formosa, una de las provincias más pobres del país. En su presentación, Zamba viaja en el tiempo e interviene en la Revolución de Mayo.

En seguida se produjo la creación y la puesta al aire de la señal infantil Pakapaka, dependiente del Ministerio de Educación de la Nación, donde Zamba, a la vez que apareció ubicado como el principal producto, fue cosechando polémicas valoraciones. El programa fue reconocido por diversos sectores del periodismo cultural y por diferentes intelectuales como un producto masivo y exitoso, lo que parecería traducirse no solo en reconocimientos locales e internacionales, ${ }^{3}$ sino en que La asombrosa excursión de Zamba, primero, y Mundo Zamba, más tarde, aparecían como una opción que disputaba públicos en la oferta de productos culturales infantiles. Esa ampliación de públicos no solo se plasmó en estrategias desde la oferta de contenidos sino otras acciones tales como la disputa legal con el grupo Clarín, del que forma parte la empresa Cablevisión, para que cumpliera con la Ley Nacional 26.522 e incluyera el canal Pakapaka en su grilla. Después de tres años y medio, en 2014, la justicia obligó al multimedios a cumplir esta normativa que fue dejada nuevamente sin efecto por el nuevo gobierno en 2018.

\section{Algunas referencias sobre el producto y la producción}

Con la creación y la puesta al aire del canal infantil Pakapaka, en 2010, se sumaron capitulos de Zamba sobre la Vuelta de Obligado, la Casa de Tucumán, Yapeyú, la Casa de Sarmiento, las Invasiones Inglesas, la Guerra de Malvinas y la última Dictadura cívico militar, entre muchos otros. En total, se cuentan cuatro temporadas y quince capítulos, además de algunos micros.

Para acompañar a Zamba fueron creados Niña, una nena afroamericana, y el "Niño que lo sabe todo", quien con antifaz y capa de superhéroe interactúa con el protagonista. Este personaje adivina lo que va a pasar y siempre se saca "muy bien, 10». Cada vez que comienza su explicación, Zamba exclama: 
"¡Me aburro!». Otro personaje es la señorita Silvia, la maestra de Zamba y del "Niño que lo sabe todo". Siempre camina delante de una fila de alumnos, hablando, y nunca se da cuenta cuando Zamba se escapa para viajar en el tiempo. Se suma el chico cauteloso, pelirrojo, algo pálido, con pecas y rulos, que se muestra temeroso a la hora del deporte y siempre se lastima. Sus percances son utilizados como introducción a explicaciones físicas y de salud a cargo del Dr. Ramón Carrillo. Por ultimo está Charango, un quirquincho.

Otros personajes centrales son José de San Martín, que tiene porte y capa de superhéroe, y Manuel Belgrano, figuras de gran popularidad entre el público infantil que seguía el programa. Se suman Pueyrredón y Álzaga, entre otros, y los villanos más reconocidos o malos: el Capitán realista, los militares ingleses o el Capitán anglo-francés.

Según enunciaban las autoridades del gobierno que lo produjo, este producto, en consonancia con el objetivo central de las políticas públicas implementadas entre 2010 y 2015 en materia de comunicación, buscaba fomentar la pluralidad de voces y de relatos en materia audiovisual, es decir, la democratización de la producción simbólica. En tal sentido, podemos decir que si intervenir exitosamente en el ámbito cultural implica incidir en los códigos culturales y, por lo tanto, en los comportamientos, la democratización cultural tal como fue planteada parecía apuntar a convertirse en una acción contra la hegemonía. Es decir, esa referida pluralidad de voces parecía disputar ciertas posiciones en la circulación masiva de los relatos y los enunciados históricos.

Indagamos, por lo tanto, en las valoraciones sobre una iniciativa estatal en la producción audiovisual en un ámbito históricamente dominado por el mercado. Ese fue un aspecto novedoso y desde donde se articularon, precisamente, las tensiones y las disputas de un producto que, considerado masivo, pareció haber incorporado valoraciones de ciertas posiciones contrahegemónicas en la construcción del relato histórico.

En 2015, con el cambio de gobierno, se cierra un ciclo para Zamba. A partir de 2016, este cambia su signo ideológico ${ }^{4}$ y lo comunica. No obstante, ese año la circulación dejó de tener la masividad anterior y algunas disputas del presente, vehiculizadas en el relato histórico a partir de Zamba, pueden leerse en la prensa digital. 


\section{Notas sobre la puesta en circulación}

Hasta 2013, La asombrosa excursión de Zamba contó con capítulos largos. Luego, para llevar adelante esquemas interactivos con contenidos en la web y capitulos cortos (de 5 a 10 minutos) en los que los personajes explican temáticas de interés, se pasó a Mundo Zamba, donde había digresiones sobre el cuerpo humano, la música, el arte, la paleontología y la historia de la comunicación.

Además, se incluyeron el programa participaciones de chicos/as de todo el país en y un segmento denominado "Quiero mi monumento", donde próceres y personalidades competían por un monumento. En este último esquema, en el que encontramos una clara cita y referencia a los programas televisivos de preguntas y respuestas en los que se compite por premios, se centraron muchas de las críticas que apuntaban a la contraposición de personajes de la historia como antagonistas que compiten por significados correctos en la interpretación de ciertos fenómenos. 5

Asimismo, se sumaron a la propuesta una página web y un musical que viajaba por el país. Se facilitaba a las escuelas la asistencia al musical en el parque temático Tecnópolis, en cuya plaza de juegos actuaron los personajes de los diferentes capítulos de Zamba.

Como señalamos, el producto pasó por diferentes etapas relacionadas con las decisiones sobre su circulación y su posicionamiento. Desde los inicios y hasta diciembre de 2015, la emisión de Pakapaka era de lunes a viernes, se repetía en tres horarios y el programa se emitía también por la TV pública, el canal estatal nacional. En 2014, a partir de una decisión de anclaje territorial, hubo además espectáculos en el Centro Cultural Kirchner con la banda musical y una obra teatral con los personajes de Zamba, que repitieron varias funciones que en seguida agotaron las entradas. Asimismo, empezaron a circular muñecos y disfraces de la serie, en su mayoría, producidos por cooperativas o por artesanos.

Un aspecto relevante en relación con su posicionamiento como producto masivo fue la difusión de temas musicales interpretados por músicos de amplia circulación en la industria cultural. La convocatoria a referentes que podríamos denominar de la cultura popular masiva, colaboró con la ampliación de los públicos infantiles. La banda de Zamba visitaba escuelas 
y se presentaba en diversos sitios. Pero, además, diversos referentes musicales interpretaron en otros registros marchas patrióticas o canciones del repertorio considerado del folklore nacional. Tal es el caso de la cantante de cumbia conocida como Gladys, la bomba tucumana, que cantó "Juana Azurduy", antes reconocida por la interpretación realizada por Mercedes Sosa. La Mosca, banda de rock alternativo que interpretó la marcha de San Lorenzo. El músico Horacio Fontova que interpretó un candombe sobre Artigas, entre otros. Soledad Pastorutti, la cantante que hizo masivo el folklore como género entre los más jóvenes a principios de 2000, etc. Es decir, músicos reconocidos y géneros de amplia circulación en la industria cultural con múltiples fusiones e interpretaciones híbridas.

Sumado a todo esto, o quizás precisamente por esa masividad, Zamba resultó un material de trabajo escolar efectivo y práctico para docentes de escuelas primarias, tal como surge de las entrevistas realizadas en CABA. Estos, ya sea desde lecturas oposicionales, negociadas o de total aceptación de la propuesta, casi en igual medida, empezaron a utilizarlo para introducir temas de historia y para trabajar las efemérides. "Todos los chicos lo conocen, se lo saben y eso nos hace más fácil», indicó una de las docentes entrevistadas.

A pesar de que el Ministerio de Educación envió algunos capítulos en soporte $\mathrm{CD}$, la principal vía de circulación del producto en las escuelas parece haber sido el canal de Youtube Mundo Zamba. De este modo, el esquema planeado desde el Ministerio de Educación, primero, y luego, específicamente, desde el canal Pakapaka -que lo fueron posicionando con estrategias destinadas a constituirlo como un producto masivo, en CABA y en otras ciudades del paísse sumó la institución escolar, la escuela primaria.

Sin embargo, la circulación se modificó significativamente con el cambio de gobierno y de signo político en 2016. Según enunciaban algunos/as docentes de CABA ese año siguieron utilizando el material para las efemérides escolares, pero la circulación entre el público infantil por fuera de la escuela parecería ser bastante menor. En Pakapaka pasó a emitirse solo los domingos a las 16 horas y dejó de pasarse en la televisión pública. Además, todos los espectáculos relacionados con Zamba fueron suspendidos y en Tecnópolis se desmanteló el parque temático infantil del personaje. Precisamente, en esa oportunidad tuvo lugar la difusión de fotos y de notas periodísticas que 
mostraban al muñeco del Parque destruido. Tanto en los medios hegemónicos como contra-hegemónicos, Zamba era nombrado como elemento de disputa politica e ideológica.

Para el aniversario de la independencia, el nuevo gobierno, con nuevas autoridades y nuevos asesores en historia, estrenó capítulos del personaje que fueron promocionados en los portales de los medios gráficos. El relanzamiento presentó la nueva etapa con el mismo personaje pero sin maniqueísmos o sobreinterpretación de la historia.

\section{Valoraciones de la prensa:}

\section{Zamba considerado un producto masivo hasta 2015}

Si bien entre 2010 y 2015 hubo algunas variaciones respecto de las valoraciones que circularon en la prensa, las cuales podrian estar asociadas tanto a las modificaciones del producto y de su circulación como a hechos políticos relacionados con este, retomamos aquí las que consideramos fueron predominantes en este periodo.

Desde la conceptualización de Valentín Volóshinov (2009 [1929]), a todo signo pueden aplicársele criterios de una valoración y el área de la ideología coincide con la de los signos. Es decir, el carácter sígnico es la determinación general de todos los fenómenos ideológicos. Todo signo ideológico al plasmarse en el proceso de comunicación social está determinado por el horizonte social de una época y de un grupo social dados.

Durante el periodo 2010/2015, La asombrosa excursión de Zamba fue considerado o definido por el periodismo cultural de los principales medios nacionales como un producto masivo. Si bien no queda claro en qué consistió tal definición de su masividad, lo cierto es que tanto Página/12, medio considerado afin al gobierno, como la prensa opositora y detractora de la política comunicacional que impulsó este producto -que, además, vio afectados sus intereses económicos por esta política, sumado a las mencionadas controversias legales-, lo construyeron como un producto masivo exitoso.

En junio de 2013, una periodista del diario La Nación emitía el siguiente juicio: 
Casi todos los adultos que tengan un hijo en la primaria lo conocen: el chico formoseño que, con sus aventuras históricas en la pantalla de Encuentro y Paka Paka, se convirtió en el primer fenómeno educativo local desde la aparición de la revista Billiken (Graña, 20/07/2013).

También en 2013, una periodista de Página/12, portal considerado por ese entonces ultra oficialista, en una nota sobre el canal infantil del Ministerio de Educación, refería a Zamba como un producto masivo valorado positivamente:

[Pakapaka] propone una perspectiva audiovisual nueva (algo así como un Baficito las 24 horas y en tu idioma) [...] Tiene sus propios líderes como Zamba, alumno nacido y criado en Formosa. "Mickey no llega a La Fiesta de la Muzzarella en La Pampa. Zamba, sí —cuenta Verónica Fiorito, directora del canal-. Desde Hijitus hasta hoy no teniamos un personaje con esta fuerza, y encima que se vista con un guardapolvo, cuente la historia de Argentina y los chicos lo sigan por eso...n. (Mansilla, 16/08/2013).

En febrero de 2014, en una noticia publicada en La Nación se admitía:

El protagonista de un dibujo animado dedicado a hacer atractiva la historia para los chicos es un éxito de la TV Pública (San Martín, 02/02/2014).

En la mayoría de los casos, la adjetivación ubica el conflicto con el producto: Zamba es polémico y causa revuelo por cómo narra la historia, es decir, aparece un juicio moral desde lo estético.

Si entendemos que cada enunciado tiene siempre una valoración o una orientación axiológica, encontramos que desde los medios y los actores que intervienen desde posiciones hegemónicas Zamba es descalificado por sus valoraciones ideológicas del relato histórico, que son ubicadas afines al gobierno, frente a una neutralidad que sería lo valorado positivamente. De tal forma estaría nombrada la polémica.

No obstante, no podemos dejar de advertir que tanto Pakapaka como Zamba iniciaron una disputa desde una posición de legitimación cultural. Al equiparar al canal con el Bafici, el festival de cine independiente más importante de la Argentina, la directora de Pakapaka situaba y dejaba translucir que se trataba 
de un producto masivo que, a la vez, se podía posicionar desde cierta expresión cercana a una vanguardia. ${ }^{6}$ En el enunciado aparece una valoración social que nos ofrece algunas pistas sobre la singularidad de esta expresión cultural: un producto masivo que pone en juego un posicionamiento de relativa autonomía y de reconocimiento positivo en el campo de la cultura.

\section{Entre la historia y la estética}

En agosto de 2012, con el título "Un dibujito de Paka Paka cuenta la "historia oficial" en versión infantil», la sección sociedad del diario La Nación publicó una extensa nota en la que se consultaba la opinión de distintos/as especialistas.

En primer término, nos detenemos en la calificación de oficial. Aquí, el adjetivo cumple la función de transmutar el sentido. Si Zamba viene a contar otra versión de la historia, la anterior sería la oficial. Sin embargo, el título del diario que históricamente representa a los sectores de poder económico de la Argentina, construye como oficial lo que viene ideológicamente desde el gobierno que gestiona el Estado, equiparando, de este modo, oficialismo político con oficial como lo instituido.

En la nota se cita como voz social autorizada a un intelectual por su función como tal y como padre.

\footnotetext{
Marcos Novaro es sociólogo y doctor en Filosofia, pero también es padre de tres chicos. Ya estaba familiarizado con las aventuras de Zamba al momento de la entrevista que le hizo La Nación, y desde un primer momento expresó preocupación por la serie. "La escuela no puede dar productos de tan poca calidad, que tienen tantos defectos y que encima transmiten formas de pensar", opinó (Struminger, 03/08/2012).
}

Encontramos en esto una regularidad que se repite: la descalificación estética, por un lado, y la pretensión de neutralidad, por otro. Es decir, como si fuera posible concebir los enunciados sin valoración axiológica.

La nota continúa con otra experta que legitima las valoraciones sobre el producto: 
[...] Zamba fue creado bajo la órbita del Ministerio de Educación. En esa distinción se basó la doctora en Comunicación Pública, Gabriela Fabbro, para examinar el dibujo animado.

La especialista en medios sostuvo que la responsabilidad de los canales que responden al Estado es mayor de la que poseen las señales privadas. "La televisión estatal es un servicio público con intenciones didácticas, una institución más allá del partido gobernante. Este gobierno utiliza el potencial que tiene la TV pública para transmitir ideología, y esto es una tergiversación de la función que debe tener", señaló (Struminger, 03/08/2012).

Nuevamente, aparece una concepción de neutralidad como lo esperable desde el Estado, que borra las posiciones y las condiciones materiales de la enunciación. La pretensión de la comunicación estatal como servicio público, algo que no parece ser considerado como tal para las señales privadas.

Por su parte, la directora de Pakapaka, Cielo Salviolo, defendió el trabajo realizado en el ciclo infantil y subrayó que "cualquier relato histórico parte de una mirada particular, situada». En una entrevista con La Nación vía correo electrónico, la funcionaria expresó: "Siendo un canal público tenemos la responsabilidad de no interpelar a los chicos y chicas como consumidores, de respetar sus identidades, sus diversidades, sus diferencias y de interpelarlos como ciudadanos" (Struminger, 03/08/2012).

La principal definición desde la cual la funcionaria sitúa la disputa es a partir de ciudadanía/consumidores. Así, los signos ideológicos que sobresalen en la confrontación parecerian ser, por un lado, la neutralidad del relato histórico frente a una noción de reconocimiento de las posiciones de enunciación. Por el otro, las valoraciones sobre el Estado y sus medios. Si para la especialista el Estado debe cumplir una función didáctica neutral, para la funcionaria tiene que concebir a la infancia como ciudadanía en oposición a consumidores.

La nota refuerza la importancia de la neutralidad con otro especialista como voz social legitimada, desde una valoración estética. 
Fabbro, que se desempeña como directora del Observatorio de la Televisión de la Universidad Austral, coincidió con Gargarella, y añadió: «Este dibujo animado no presenta la estructura mínima de dos campanas, la otra mirada, y a veces la omisión es la peor manipulación”.

Novaro, en cambio, consideró que la serie, además de constituir un producto para el público infantil, es un "producto infantilizado". "Los chicos que lo ven pueden sacar como conclusión quiénes eran los buenos y quiénes los malos, pero nunca entender de qué se trataba el problema, qué estaba en discusión en esa época, cuál era el contexto, y eso es una reducción de la historia", enfatizó.

Por el contrario, Salviolo resaltó que el dibujo animado posibilita "llegar" mejor a los niños porque tiene códigos cercanos a ellos. "Permite jugar con lo fantástico como el paso del tiempo, pero también tiene algunas limitaciones propias, como la síntesis". Y remarcó que Zamba fue realizado con la colaboración de historiadores y la consulta de relatos académicos. "Nos parece que lo fundamental tanto para crear los personajes como el relato, es buscar fuentes diversas que permitan no reproducir estereotipos de manual o de figuritas", señaló (Struminger, 03/08/2012).

Es decir, en este período, la discusión en términos estéticos predomina en todas las posiciones. La enunciación del relato histórico desde una cierta postura ideológica parece despertar disputas en el presente que desde posiciones hegemónicas se traducen en una pretensión de neutralidad y en la descalificación estética.

Podemos inferir, desde el otro lado, una intención básica de difundir la historia entre el público infantil que, por disposiciones ideológicas del contexto, de los productores y de los agentes estatales que participaron del proyecto, promovió la tarea de revisarla a partir de un personaje que lo habilitaba.

Con la creación de Zamba, se produjo un gesto de modificar o de intervenir en los estereotipos de los personajes destinados al público infantil, a la vez que los "momentos" históricos seleccionados se relacionaban con aspectos de la historia de por sí controversiales. 


\section{La prensa digital en 2016:}

\section{de la historia y la estética a la política}

En 2016, la primera mención sobre Zamba en los portales de noticias ubicaba al producto en los conflictos y las disputas políticas del presente. La referencia, relacionada con el desmantelamiento de la plaza temática ubicada en el parque Tecnópolis, apareció cuando comenzaron a circular en las redes sociales fotos del muñeco destruido y los portales lo construyen como noticia. Lo cierto es que el hecho en las redes sociales y en los medios masivos se presentó nombrado y anclado en las controversias políticas de la actualidad.

En abril, Clarín que remitía a esto de manera directa en una volanta:

La grieta

Sacaron a Zamba de Tecnópolis y se armó la polémica

Una imagen del personaje animado de Pakapaka desmantelado disparó la reacción del kirchnerismo duro en las redes sociales. Desde el gobierno niegan que haya discriminación ideológica.

Se dice que "una imagen vale más que mil palabras". Sin dudas, la que fue difundida ayer a través de Twitter sirvió de disparador para una nueva polémica entre kirchneristas y la administración de Mauricio Macri. El muñeco que personifica a Zamba, el niño de las tiras infantiles del canal Pakapaka, en las que repasa momentos de nuestra historia junto al General San Martín, Manuel Belgrano y otros próceres, fue retratado en la basura tras el desmantelamiento de su parque temático (Clarin, 30/04/2016).

Desde otra posición ideológica, el portal del diario Página/12 también tomó el tema, aunque le otorgó menor relevancia que el medio anterior:

\section{La destrucción de Zamba}

Durante la jornada de ayer circularon por las redes sociales fotos de la imponente instalación que en Tecnópolis recreaba el personaje de Zamba y que terminó reducida a un manojo informe de hierros retorcidos. [...] "Pienso que tuvieron que serrucharla para dejarla como la dejaron", contó a este diario Fernando Salem, uno de los creadores del popular personaje 
junto con Sebastián Mignona, Nicolás Dardano y un gran equipo multidisciplinario que trabajó a las órdenes del Ministerio de Educación. $\mathrm{El}$ artista manifestó que el hecho le provocó "sorpresa" y que "ver destruido el trabajo de años le pareció doloroso" (Página/12, 29/04/2016).

El producto, llegado a este punto de cambio de gobierno y de signo ideológico, aparecía de uno y otro lado como elemento condensador de la disputa política en términos simbólicos. En el diario opositor al gobierno que lo implementó esto se presentó más destacado, con una noticia notablemente más extensa. Así aparecía también, en un sentido más literal la enunciación cuando unos meses después se anunciaba un relanzamiento del programa infantil.

A mediados de 2016, todavía fresca la experiencia de Zamba como producto masivo, el gobierno anunció el estreno de un nuevo capítulo con motivo del Bicentenario de la Declaración de la Independencia. Esto se promocionó y se difundió en los medios, donde aparecieron valoraciones que diferenciaban un antes y un después.

En mayo, El Cronista tituló en su portal:

El gobierno relanzará el dibujito Zamba:

"Fue la primera victima del kirchnerismo"

Así lo adelantó Hernán Lombardi, titular del Sistema Federal de Medios y Contenidos Públicos. Si le marcas un discurso único Zamba es una víctima. En los nuevos capítulos históricos que pueda tener otras miradas, analizó. Lombardi ironizó: "A mí no se me ocurre que Zamba curioso vaya a la Patagonia a ver los autos de Lázaro (Báez), nosotros no somos asi” (El Cronista, 13/05/2016).

Ya no aparece tematizada la evaluación estética. Las valoraciones son politicas y coyunturales. El funcionario hacía referencia, directamente, a un hecho de la política contemporánea desde donde se construye al adversario a partir de la asunción del nuevo gobierno: la disputa moral a partir de la lucha contra la corrupción del adversario derrotado. Es decir, si desde el inicio el nuevo gobierno centró su construcción discursiva en la corrupción del gobierno anterior que venia a castigar, Zamba aparece ubicado como un elemento neutral que ahora podría narrar la historia desde el signo contrario. Solo que 
ese signo contrario, más que serlo respecto a la historia, es nombrado por el periodismo opositor en relación con el presente de la política.

Otro aspecto es la definición del personaje en su etapa anterior: discurso único.

Esto también puede constatarse en la sección espectáculos de Infobae, que en julio anunciaba:

\section{"Zamba" vuelve a la televisión "sin sobreinterpretación"}

El dibujo animado regresa a la pantalla de Pakapaka para celebrar el Bicentenario. Para Gabriela Ricardes, secretaria de Contenidos de la Nación, el personaje "había perdido su curiosidad inicial por la historia y empezado a formar parte del relato del poder" (Infobae, 07/07/2016).

En julio, en el portal de noticias Big Bang News se hacia de la siguiente manera:

Zamba cierra la grieta: de los destrozos a su regreso en TV

La serie de la señal infantil Paka Paka volverá mañana para conmemorar el Bicentenario de la Independencia. El programa se repetirá a lo largo del día y tendrá como premisa alejarse de las interpretaciones políticas (Big Bang News, 08/07/2016).

En este punto, es interesante destacar que el poder aparece equiparado, tanto aquí como a lo largo de todo el periodo analizado, al gobierno. Es decir, el poder es para los diarios opositores el poder politico del gobierno a partir de la legitimidad de los votos. Pero no parece ser el poder económico. El poder se nombra solo como sinónimo del sector político que gobierna.

La valoración estética, para entonces, ha desaparecido. Lo que se nombran son los efectos políticos del anterior relato histórico en el presente.

\section{Apropiaciones y valoraciones docentes}

Como señalamos, La asombrosa excursión de Zamba se posicionó en la oferta de productos culturales infantiles disputando públicos a otros productos masivos, ${ }^{7}$ con códigos culturales hegemónicos y desde una política pública. 
En los medios de comunicación, sin embargo, Zamba aparece nombrado como lo hegemónico. Esto es claro cuando se lo describe como discurso único. Frente a esto, indagamos a partir de entrevistas cómo se materializaron esas disputas para los/as docentes, uno de los públicos que más se apropió del producto, ya sea desde lecturas oposicionales, negociadas o dominantes.

La noción de apropiación, concebida como el proceso material y simbólico de asignación y de interpretación de sentidos a un producto cultural, pone el énfasis en la capacidad de los sujetos para volver significativos los procesos culturales, de acuerdo a sus propios propósitos y contextos.

Lo analizado, permite visualizar que los/as docentes hacen diversas apropiaciones del producto audiovisual en cuestión a partir de usos más o menos similares. Esto es, los diferentes marcos materiales y simbólicos importan sentidos disimiles para los actores que incorporan significados, imágenes y narrativas provenientes de textos audiovisuales que son mediados por condiciones específicas de la experiencia vivida. De manera tal que los sentidos no se condicen necesariamente con la producción mediática, sino que más bien se establecen complejos vínculos de retroalimentación, de proximidad y de distancia (Hall, 1980).

Cabe señalar en este punto, que si bien las valoraciones aparecen en relación con el uso como docentes el producto no fue pensado con fines didácticos.

Ahora bien, las primeras valoraciones respecto del producto surgen, tal como señalamos en el caso de la prensa, desde el juicio estético. En general, son lecturas negociadas, donde se rescata la iniciativa de un personaje local y la tematización de la historia, aunque en relación con esta última aparece una valuación negativa desde lo estético que en la mayoría de los casos se vincula al género dibujo animado.

Con respecto a la historia, me gusta. Lo que no me gusta es el formato, le quita credibilidad. Lo hace más infantil (...) le saca la oportunidad de ver que es real la historia. (...) lo primero que no me cerró es por qué eligieron el formato dibujito. Y el estereotipo (Laura, docente de escuela pública, CABA). 
A mí no me va lo del héroe. La idea de que sea un tipo con determinados valores, que se la jugó en determinadas situaciones, ¿héroe? Hay un montón de héroes. En el dibujito están muy satirizados (Ángela, docente de escuela pública, CABA).

A mi criterio, Zamba es un material, un recurso, que hay que usarlo con alguien porque el chico, es verdad, mira la información y sabe algo, pero sabe una parte, no sabe todo, a veces ridiculizan cosas. A un chico le sirve, pero es como una historieta de malos y buenos y no somos malos y buenos. Además, no muestra qué pasaba realmente en ese lugar. Arma un enfoque (Silvina, docente de escuela pública, CABA).

Ese juicio estético parece estar en consonancia con las valoraciones del periodismo y de los/as expertos: estereotipos, muy infantil, satirización de los héroes, arma UN enfoque. El otro aspecto es el que, tal como señalamos, tiene más relevancia en los medios en 2016: la historia como disputa política en el presente. Sin embargo, esta aparece en detrimento del juicio crítico. "El tratamiento de la historia es la politica misma. La hacen los que ganan. Apunto al juicio crítico. El dibujito no lo propicia tanto" (Andriu, docente de escuela pública, CABA).

Es decir, si a prima facie el producto Zamba incorpora un punto de vista de la historia que sería afin a posiciones ideológicas que se postulan vinculadas a la ideología del gobierno, esto implica pensar que se contrapone a otros puntos de vista con los que viene a discutir y que serian los instituidos. Si entendemos con Voloshinov (2009) que todo signo es ideológico, advertimos que cada uno de los productos culturales infantiles resultan tales. Sin embargo, la naturalización de los signos hegemónicos refuerza e impide advertir esto último entre los/as docentes entrevistados/as.

En relación con esto, podriamos establecer, además, alguna relación con la amplia circulación por parte de las voces legitimadas de expertos y del periodismo de un signo ideológico acorde a lo que mencionamos como la definición del poder. Este es nombrado y reforzado desde el sentido común en los medios masivos como el poder político del gobierno. Se aloja ahí. Si el producto Zamba plantea una postura ideológica esta es la del poder, porque es un producto del Estado, con una gestión de gobierno. 
Por último, interesa destacar otro de los aspectos anteriormente señalado. Zamba aparece como un producto que disputa desde los signos ideológicos algunos aspectos de la hegemonía en relación con dimensiones residuales de la historia. Lo hace desde una estrategia que lo ubica como un producto de circulación masiva, pero con la particularidad de ubicarse en una posición legitimada en el campo de productos culturales. Este aspecto, que ubicamos anteriormente respecto de las valoraciones de los medios, aparece también entre las valoraciones docentes.

Las familias súper opositoras o gorilas ponen Paka paka. Creo que ahí hubo un acierto de superación de lo partidario y Paka paka, encuentro y Zamba quedaron como buenos productos culturales más allá de donde vengan (Joaquín, docente de escuela pública, CABA).

\section{Algunas conclusiones}

Indagamos en este trabajo acerca de la circulación de significaciones y de valoraciones sobre La asombrosa excursión de Zamba, de 2010 a 2016. Por un lado, entre actores legitimados en el campo intelectual, periodismo y referentes académicos consultados como expertos por los medios de comunicación; por otro, analizamos algunas de las valoraciones desde los usos y las apropiaciones de docentes. Estas entrevistas, realizadas durante 2016, nos permitieron rastrear en la circulación algunas valoraciones entre actores que, haciendo un uso específico de este producto cultural, realizaron diferentes lecturas, en su mayoria negociadas (Hall, 1980) y en un contexto de cambio político.

La serie, que se autodefinía y que se fue constituyendo como una comunidad en línea, propuso contenidos culturales desde una política pública de intervención en el campo simbólico a partir del relato histórico en una línea que, a medida que avanzaba, parecía generar cada vez más controversias en el presente.

En tal sentido, uno de los aspectos del enfoque aquí adoptado es la pregunta por la politicidad en la cultura. Es decir, los interrogantes que se desprenden de los modos en que se organiza y se disputa simbólicamente la vida en común, en el espacio público donde la historia ocupa un relevante lugar en el 
presente. Esto último, entendido en relación con elementos residuales de la cultura, esto es, aquellos que dan cuenta de lo que proviniendo del pasado todavia se halla en actividad dentro del proceso cultural y es activado en el presente (Williams, 2000).

Como decíamos al inicio, partimos de la hipótesis de que los sentidos no se condicen necesariamente con la producción mediática, sino que más bien se establecen complejos vínculos de retroalimentación, de proximidad y de distancia (Hall, 1980).

Tanto en las valoraciones de los medios digitales seleccionados para el análisis como en las de los/as docentes de CABA, encontramos en su mayoría lecturas oposicionales y/o negociadas donde los signos ideológicos controversiales parecen ser, en primer término, las nociones de neutralidad o no frente a los discursos mediáticos relacionados con la historia.

En relación con esto último, que aparece enunciado de diferentes maneras, ya sea en términos literales o como valoración estética, no podemos perder de vista ciertas disimetrias: la puesta en circulación de significaciones de la prensa digital sobre el producto Zamba parece haber establecido ciertas mediaciones comunicacionales en relación con los significados históricos, ideológicos, políticos y estéticos en los usos y las apropiaciones de los/as docentes.

Se suman a esto otras pistas sobre la singularidad de esta expresión cultural: un producto masivo que pone en juego un posicionamiento de relativa autonomía y de reconocimiento positivo en el campo de la cultura, pero que activa conflictos desde elementos residuales de la cultura a partir de la disputa de ciertos signos ideológicos (Volóshinov, 2009 [1929]) que pone en circulación.

\section{Referencias}

Bourdieu, P. (1991). El sentido práctico. Madrid, España: Taurus.

Comité Federal de Radiodifusión (COMFER). (2009). Informe Contenidos de la Televisión Abierta Argentina. Origen de la Producción - Tipo de Programas - Estructura de Propiedad. 
Grossberg, L. (2006). Stuart Hall sobre raza y racismo: estudios culturales y la práctica del contextualismo.

Tabula Rasa, (5), 47-54. Recuperado de https:/ / revistas.unicolmayor.edu.co/index.php/tabularasa/ article/view/ 1588

Guber, R. (2009) [1991]. El salvaje metropolitano.

Ciudad Autónoma de Buenos Aires, Argentina: Paidós.

Hall, S. (1984). Notas sobre la deconstrucción de lo popular. En R. Samuels (Ed.), Historia popular y teoria socialista (pp. 93-112). Barcelona, España: Crítica.

Hall, S. (1980). Culture, Media and Language.

Londres, Inglaterra: Hutchinson.

Volóshinov, V. N. (2009) [1929]. El marxismo y la filosofía del lenguaje (Trad. de Tatiana Bubnova).

Ciudad Autónoma de Buenos Aires, Argentina: Godot.

Williams, R. (2000). Marxismo y literatura.

Barcelona, España: Biblos.

\section{Fuentes}

Entrevistas a docentes de CABA en 2016.

Entrevista a realizadores en 2016.

Big Bang News (8 de julio de 2016). Zamba cierra la grieta: de los destrozos a su regreso en TV. Recuperado de https:/ / www. bigbangnews.com/politica/zamba-cierra-la-grietade-los-destrozos-a-su-regreso-en-tv-2016-7-8-15-36-0 
Clarín (30 de abril de 2016). Sacaron a Zamba de Tecnópolis y se armó la polémica. Recuperado de https:/ / www.clarin.com/politica/zamba-medio-polemicamacristas-kirchneristas_0_N1Di8_hlZ.html

El Cronista (13 de mayo de 2016). El gobierno relanzará el dibujito Zamba: "Fue la primera víctima del kirchnerismo». Recuperado de https://www.cronista.com/economiapolitica/El-Gobiernorelanzara-el-dibujito-Zamba-Fue-la-primera-victima-delkirchnerismo-20160513-0104.html

Graña, D. (20 de julio de 2013). Televisión. El chico que puede cambiar la pantalla. La Nación. Recuperado de https://www.lanacion.com.ar/espectaculos/television/el-chicoque-puede-cambiar-la-pantalla-nid1602603/

Infobae (7 de julio de 2016). "Zamba" vuelve a la televisión "sin sobreinterpretación". Recuperado de https:/ / www.infobae.com/teleshow/infoshow/2016/07/07/zam ba-vuelve-a-la-television-sin-sobreinterpretacion/

Ley Nacional 26.522 de Servicios de Comunicación Audiovisual (2009). Recuperado de

http:/ / servicios.infoleg.gob.ar/infolegInternet/anexos / 155000 159999/158649/norma.htm

Mansilla, M. (16 de agosto de 2013). Ojos nuevos. Página/ 12. Recuperado de

https: / / www.pagina12.com.ar/diario/suplementos/las12/138244-2013-08-16.html

Página/12 (29 de abril de 2016). La destrucción de Zamba. Recuperado de https://www.pagina12.com.ar/diario/economia/2-298124-201604-29.html 
San Martín, R. (2 de febrero de 2014). Zamba: historia nac\&pop para principiantes. La Nación. Recuperado de https:/ / www.lanacion.com.ar/opinion/zamba-historia-nacpoppara-principiantes-nid1660556/

Struminger, B. (3 de agosto de 2012). Un dibujito de Paka Paka cuenta la "historia oficial" en versión infantil. La Nación.

Recuperado de https:/ /www.lanacion.com.ar/sociedad/zamba-depaka-paka-relata-la-historia-oficial-en-version-infantil-nid1495635/

Struminger, B. (19 de marzo de 2015). Jug.Ar, el plan del Gobierno para fabricar juguetes y remeras Nac\&Pop. La Nación. Recuperado de https:/ / www.lanacion.com.ar/politica/jugar-el-plan-delgobierno-para-fabricar-juguetes-y-remeras-nacpop-nid1772963/

\section{Notas}

1 Investigación realizada por la autora en el marco de su Tesis de Doctorado "La circulación de La Asombrosa excursion de Zamba: valoraciones acerca de la historia política" para el Doctorado en Ciencias Sociales de la Universidad de Buenos Aires.

2 Nos referimos a la noción de campo bourdiana (Bourdieu, 1991), aunque no en sentido estricto.

3 En 2015, resultó nominado con el capítulo sobre la última Dictadura cívico militar en el rubro "Animación infantil" del International Kids Emmy Awards, uno de los principales certámenes que entrega la industria de la televisión mundial.

4 Nos referimos al concepto de Volóshinov (2009 [1929]), para quien las conciencias individuales están ligadas entre sí por los signos, que solo aparecen en la interacción, en lo que él llama la "cadena ideológica", por lo que "la conciencia no puede realizarse sino es en el material la encarnación semiótica" (p. 68).

5 Así surge, también, de las entrevistas realizadas a integrantes de la producción.

6 Cuando nos referimos a vanguardia lo hacemos a partir de una noción posicional del término, en relación con el carácter precursor o, en términos bourdianos (Bourdieu, 1991), por su caracter de mayor autonomía en el campo de la producción cultural. 
7 Según un informe del Comité Federal de Radiodifusión (COMFER) de diciembre de 2009, el porcentual de programación dedicado al público infantil era muy bajo. Hasta entonces, la totalidad de la programación infantil proveniente del exterior era de origen norteamericano y superaba a la programación nacional en todas las regiones entre 35 y 50\%. En la región AMBA se emitía 42,85\% más de programación infantil con respecto al resto del país y la retransmisión en cada región del interior era mayor que la producción local, lo que establece la hegemonía que el AMBA ejerce sobre el resto del país. 